\title{
Mindestens so wirksam wie Beta-Interferone, aber mehr Nebenwirkungen?
}

Fragestellung: Ist Azathioprin als Sekundärprophylaxe der schubförmigen Multiplen Sklerose (RRMS) mindestens genauso wirksam wie Beta-Interferone?

Hintergrund: Das Immunsuppressivum Azathioprin wurde seit den 1960er-Jahren zur schubprophylaktischen Therapie der RRMS eingesetzt. Eine kleine randomisierte Vergleichsstudie über ein Jahr legte nahe, dass Azathioprin klinisch eine stärkere sekundärprophylaktische Wirksamkeit besitzt als Beta-Interferone [1].

Patienten und Methodik: In diese von der italienischen Arzneimittelagentur finanzierte, zweijährige, multizentrische NichtUnterlegenheitsstudie wurden insgesamt 150 Patienten

Massacesi L, Tramacere I, Amoroso $S$ et al. Azathioprine versus beta interferons for relapsingremitting multiple sclerosis: a multicentre randomized noninferiority trial. PLOS ONE 2014; 9(11): e113371 mit aktiver RRMS eingeschlossen. Unverblindet erhielten sie randomisiert entweder eines der zugelassenen Interferon-beta-Präparate nach Wahl des behandelnden Neurologen $(n=73)$ oder
Azathioprin $3 \mathrm{mg} / \mathrm{kg} /$ Tag mit Dosisanpassung nach dem Differenzialblutbild $(n=77)$. Neurologische Untersuchungen und MRT-Auswertungen erfolgten verblindet. Primärer Endpunkt war der Schubratenvergleich über zwei Jahre.

Ergebnisse: Insgesamt 127 Patienten (84\%) schlossen die Studie $\mathrm{ab}$, jedoch brachen $39 \%$ der Azathioprin- und $26 \%$ der Interferon-Patienten die Behandlung ab oder begannen diese gar nicht erst. Das Schubratenverhältnis betrug unter Azathioprin gegenüber den Beta-Interferonen 0,67 (einseitiges 95\%-Konfidenzintervall $[\mathrm{KI}] 0,96, \mathrm{p}<0,01$ ) im Sinne einer Nicht-Unterlegenheit. In der MR-tomografisch untersuchten Subgruppe lag die jährliche Rate neuer T2-Läsionen unter Azathioprin bei 0,76 (95\%-KI $0,61-0,95 ; \mathrm{n}=50)$ und unter Beta-Interferonen bei 0,69 (95\%-KI $0,54-0,88 ; n=47 ; p=0,75)$. Therapieabbrüche wegen Nebenwirkungen waren unter Azathioprin häufiger als unter Beta-Interferonen $(20,3 \%$ vs. $7,8 \%, p=0,03)$, auch wurden unter Azathioprin insgesamt mehr Nebenwirkungen dokumentiert $(\mathrm{p}<0,01)$.

Schlussfolgerungen: Azathioprin ist eine nicht weniger effektive, aber nebenwirkungsreichere Therapiealternative zu BetaInterferonen.

\section{- Kommentar von Mathias Buttmann, Würzburg}

\section{Verdienstvolle, aber zu späte und zu schwache Antworten auf alte Fragen}

Auf Basis weniger, sehr kleiner placebokontrollierter Studien wurde Azathioprin 2000 in Deutschland als sekundärprophylaktische Reservebehandlung der RRMS zugelassen. Doch wie wirksam, verträglich und sicher ist dieses kostengünstige orale Immunsuppressivum im Vergleich zu anderen zugelassenen Medikamenten?

Die hier vorgestellte, mit öffentlichen Geldern finanzierte Studie legt ebenso wie eine vorausgegangene kleine randomisierte Studie [1] nahe, dass die schubprophylaktische Wirksamkeit von Azathioprin zumindest nicht schlechter als die von $\beta$-Interferonen ist. Allerdings war die Studie mit insgesamt 150 Teilnehmern klein und nur teilverblindet, zudem brachen sehr viele Patienten die Therapie ab - und dies zwischen den Behandlungsarmen ungleich verteilt. In der Interferongruppe erhielten nur $17 \%$ eines der hochdosierten subkutanen Interferonpräparate, die in Head-to-head-Studien anderen Interferonpräparaten in der Wirksamkeit teilweise überlegen waren. Azathioprin zeigte sich nebenwirkungsreicher als die Beta-Interferone.

Mit Teriflunomid, Dimethylfumarat und Fingolimod sind inzwischen orale Sekundärprophylaktika zugelassen, die in großen, placebokontrollierten Studien hinsichtlich ihrer Wirkungen und Nebenwirkungen wesentlich besser als Azathioprin charakterisiert sind. Auch wenn belastbare Daten zu deren Langzeitsicherheit in der MS-Therapie noch fehlen und (weitere) Wirksamkeitsvergleiche mit aktiven Substanzen zu wünschen sind, ist damit der Einsatz des myelotoxischen und karzinogenen Azathioprin [2] trotz seiner anzunehmenden Wirksamkeit weitgehend obsolet geworden.
Referenzen:
1. Etemadifar M et al. J Neurol 2007; 254: 1723-28
2. Confavreux C et al. Neurology 1996; 46: $1607-12$

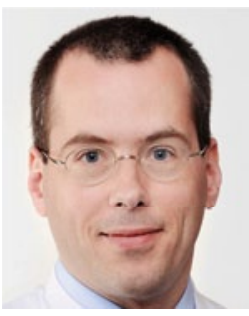

PD Dr. med. Mathias Buttmann, Würzburg

Leiter der Neuroimmunologischen Spezialambulanz, Neurologische Klinik und Poliklinik, Universitätsklinikum Würzburg E-Mail:m.buttmann@ukw.de 